\title{
Effects of coated capillary column, derivatization, and temperature programming on the identification of Carica papaya seed extract composition using GC/MS analysis
}

\begin{abstract}
Some of the common practices to assess the composition of plant extract, including Carica papaya seed extract (CPSE) are direct injection of the extract, compound separation using polyethylene glycol capillary column (DB-WAX), and non-linear-temperature programming (NLTP) of GC/MS analysis. This study specifically compared the coating of capillary column, sample derivatization, and temperature programming of GC/MS to determine the composition of CPSE. The retention indices (RI) of the detected compounds were determined and compared to the reference RI. In particular, 5\% phenyl-95\% methylpolysiloxane (HP-5MS)-, DB-WAX, and biscyanopropyl polysiloxane (HP-88)-coated capillary columns were used to identify the composition of CPSE. For this study, HP-5MS column, which separated the highest number of compounds (26 compounds) from CPSE, was deemed as the most suitable column. The GC/MS analysis of derivatized CPSE identified 21 compound groups, where fatty acids and fatty acid methyl esters served as the major compounds (80.23\%), followed by these compounds in decreasing order: amides $>$ nitriles $>$ sterols $>$ fatty aldehydes $>$ organic acids. A stronger correlation determination between the carbon number and alkane retention time of lineartemperature programming (LTP) $(\mathrm{R} 2=0.9859)$ was found, as compared to its correlation determination with NLTP $(\mathrm{R} 2=0.9175)$, which exhibited an almost equal RI of LTP to the reference RI. Conclusively, GC/MS analysis for the derivatized CPSE using HP-5MS column separation and LTP is highly recommended.
\end{abstract}

Keyword: Capillary column; Carica papaya seed; GC/MS; Sample derivatization; Temperature programming 